\title{
Combined Use of Multiple Intravascular Imaging Techniques in Acute Coronary Syndrome
}

\author{
Takashi Kubo ${ }^{1,2 *}$, Kosei Terada ${ }^{1}$, Yasushi Ino ${ }^{1,3}$, Yasutsugu Shiono ${ }^{1}$, Shengxian Tu ${ }^{4}$, \\ Tien-Ping Tsao ${ }^{5}$, Yundai Chen ${ }^{6}$ and Duk-Woo Park ${ }^{7}$ \\ ${ }^{1}$ Department of Cardiovascular Medicine, Wakayama Medical University, Wakayama, Japan, ${ }^{2}$ Department of Cardiovascular \\ Medicine, Naga Municipal Hospital, Kinokawa, Japan, ${ }^{3}$ Department of Cardiovascular Medicine, Shingu Municipal Hospital, \\ Shingu, Japan, ${ }^{4}$ School of Biomedical Engineering, Biomedical Instrument Institute, Shanghai Jiao Tong University, \\ Shanghai, China, ${ }^{5}$ Division of Cardiology, Heart Center, Cheng Hsin General Hospital, Taipei, Taiwan, ${ }^{6}$ Department of \\ Cardiology, Chinese PLA General Hospital, Beijing, China, ${ }^{7}$ Division of Cardiology, Asan Medical Center, University of Ulsan \\ College of Medicine, Seoul, South Korea
}

OPEN ACCESS

Edited by:

Jinwei Tian,

The Second Affiliated Hospital of Harbin Medical University, China

Reviewed by: Kensuke Nishimiya, Tohoku University, Japan Suma Victor,

Madras Medical Mission, India

*Correspondence: Takashi Kubo

kubo.takashi@yahoo.com

Specialty section:

This article was submitted to

Cardiovascular Imaging,

a section of the journal

Frontiers in Cardiovascular Medicine

Received: 29 November 2021 Accepted: 20 December 2021

Published: 17 January 2022

Citation:

Kubo T, Terada K, Ino Y, Shiono Y, Tu S, Tsao T-P, Chen Y and Park D-W

(2022) Combined Use of Multiple Intravascular Imaging Techniques in Acute Coronary Syndrome. Front. Cardiovasc. Med. 8:824128.

doi: 10.3389/fcrm.2021.824128
Recent advances in intravascular imaging techniques have made it possible to assess the culprit lesions of acute coronary syndrome (ACS) in the clinical setting. Intravascular ultrasound (IVUS) is the most commonly used intravascular imaging technique that provides cross-sectional images of coronary arteries. IVUS can assess plaque burden and vessel remodeling. Optical coherence tomography (OCT) is a high-resolution (10 $\mu \mathrm{m})$ intravascular imaging technique that uses near-infrared light. OCT can identify key features of atheroma, such as lipid core and thin fibrous cap. Near-infrared spectroscopy (NIRS) can detect lipid composition by analyzing the near-infrared absorption properties of coronary plaques. NIRS provides a chemogram of the coronary artery wall, which allows for specific quantification of lipid accumulation. These intravascular imaging techniques can depict histological features of plaque rupture, plaque erosion, and calcified nodule in ACS culprit lesions. However, no single imaging technique is perfect and each has its respective strengths and limitations. In this review, we summarize the implications of combined use of multiple intravascular imaging techniques to assess the pathology of ACS and guide lesion-specific treatment.

Keywords: acute coronary syndrome, plaque rupture, plaque erosion, calcified nodule, intravascular ultrasound, optical coherence tomography, near-infrared spectroscopy

\section{INTRODUCTION}

Several autopsy studies have revealed the pathology of culprit lesions of acute coronary syndrome (ACS). ACS is caused by three main mechanisms: plaque rupture, plaque erosion, and calcified nodule. Plaque rupture is the most common (55-60\%) mechanism for ACS (1). Plaque rupture usually has an extensive lipid core containing large numbers of cholesterol crystals and a thin fibrous cap $(<65 \mu \mathrm{m})$ infiltrated by foamy macrophages. Plaque erosion is found in $30-35 \%$ of ACS (1). Most of plaque erosion occurs over areas of intimal thickening, with minimal or no evidence of a lipid. Calcified nodule is identified in $2-7 \%$ of ACS (1). Calcified nodule is a plaque with luminal thrombi showing calcific nodules protruding into the lumen through a disrupted thin fibrous cap.

Recent advances in intravascular imaging techniques have made it possible to assess the culprit lesions of ACS in the clinical setting. In the early 1990s, intravascular ultrasound (IVUS) became 
clinically available, enabling morphological evaluation of coronary artery walls. In the mid-2000s, optical coherence tomography (OCT) was developed and its high resolution allows for more precise observations of coronary atherosclerosis. In the late 2000s, near-infrared spectroscopy (NIRS) was clinically applied, which permits to diagnose plaque composition. These intravascular imaging techniques can depict histological features of plaque rupture, plaque erosion, and calcified nodule in ACS culprit lesions (2).

\section{IVUS}

IVUS is the most commonly used intravascular imaging technique in the world. IVUS provides cross-sectional images of coronary arteries. IVUS can identify plaque rupture and calcified nodule, but not plaque erosion. Plaque rupture is identified as ulceration with a recess in the plaque beginning at the luminalintimal border $(3,4)$. The lesions with plaque rupture usually have a large plaque burden with positive vascular remodeling and exhibit a hypoechoic plaque with acoustic shadow derived from the lipid core (so-called attenuated plaque) (5). The coarseresolution (100-200 $\mu \mathrm{m}$ ) of IVUS cannot determine the presence or absence of small rupture, therefore this technique cannot diagnose plaque erosion. Calcified nodule is identified by a convex shape of the luminal surface with a bright echo, bulgy shape, irregular surface, and acoustic shadowing (6). The lesions with calcified nodule often have an extensive calcium sheet.

\section{OCT}

OCT is a high-resolution $(10 \mu \mathrm{m})$ intravascular imaging technique that uses near-infrared light. OCT is the most reliable technique for evaluating the complex morphology of ACS culprit lesions, although this technique requires some complicated procedures such as intracoronary contrast injection to remove red blood cells for image acquisition. OCT can identify lipid core (characterized by signal-poor regions with diffuse borders), thin fibrous cap (characterized by homogeneous, signal-rich regions), and calcification (characterized by well-delineated, signal-poor regions with sharp borders) in atherosclerotic plaques (7). Plaque rupture is defined as a disruption of thin fibrous cap with a clear cavity formed inside the plaque (8). The lesions with plaque rupture often have macrophages (defined by signal-rich, distinct or confluent punctuate regions with shadowing), vasa vasorums (defined by signal-poor, well-delineated voids within plaque), cholesterol crystals (defined as thin, linear regions of high signal intensity within the lipid plaque), and healed plaques (defined as plaques with 1 or more layers with different optical density) $(9,10)$. Plaque erosion is identified by the presence of attached thrombus overlying an intact (i.e. absence of fibrous cap disruption) plaque without superficial lipid or calcification (11). The absence of endothelial cells is a key pathological criterion for erosion, however the resolution of current OCT cannot detect individual endothelial cells. Since the OCT metrics of plaque erosion are different from the pathological definition, the term "OCT-derived erosion" is used instead of erosion.
TABLE 1 | Diagnostic criteria for plaque rupture, erosion, and calcified nodules.

\begin{tabular}{|c|c|c|c|}
\hline & IVUS & ОСт & NIRS \\
\hline Plaque rupture & $\begin{array}{l}\text { Fibrous cap } \\
\text { disruption } \\
\text { Intra-plaque cavity } \\
\text { Attenuated plaque }\end{array}$ & $\begin{array}{l}\text { Fibrous cap } \\
\text { disruption } \\
\text { Intra-plaque cavity } \\
\text { Large lipid plaque } \\
\text { Macrophages } \\
\text { Vasa vasorum } \\
\text { Cholesterol } \\
\text { crystals } \\
\text { Healed plaque }\end{array}$ & $\begin{array}{l}\text { High maxLCBI } \\
4 \mathrm{~mm}(>400)\end{array}$ \\
\hline Plaque erosion & & $\begin{array}{l}\text { Intact fibrous cap } \\
\text { Fibrotic plaque }\end{array}$ & $\begin{array}{l}\text { Low maxLCBI } \\
4 \mathrm{~mm}(<400)\end{array}$ \\
\hline Calcified nodule & $\begin{array}{l}\text { Convex calcium } \\
\text { Large } \\
\text { calcium sheet }\end{array}$ & $\begin{array}{l}\text { Convex calcium } \\
\text { Large } \\
\text { calcium sheet }\end{array}$ & $\begin{array}{l}\text { Moderate maxLCBI } \\
4 \mathrm{~mm}\end{array}$ \\
\hline
\end{tabular}

IVUS, intravascular ultrasound; $L C B$, lipid core burden index; NIRS, near-infrared spectroscopy; OCT, optical coherence tomography.

Calcified nodule is defined by fibrous cap disruption detected over a calcified plaque characterized by protruding calcification (11). Most of calcified nodule have superficially located large calcification. In ACS culprit lesions, a large amount of thrombus may cause a strong attenuation of the near-infrared light and interfere with accurate OCT assessment of plaque morphology.

\section{NIRS}

NIRS can identify lipid composition by analyzing the nearinfrared absorption properties of coronary plaques. NIRS provides a chemogram of the coronary artery wall, which enables the detection of lipid core and specific quantification of lipid accumulation measured as the lipid core burden index (LCBI) and maximal LCBI over any $4 \mathrm{~mm}$ segment (maxLCBI4mm). Unlike IVUS and OCT, NIRS can accurately measure lipid component even in the presence of thrombi. NIRS is currently available as a combination catheter with IVUS. NIRS can compensate for the lack of IVUS ability to detect plaque erosion. Plaque erosion has a significantly lower maxLCBI4mm than plaque rupture and calcified nodule $(12,13)$. The optimal cutoff value for maxLCBI $4 \mathrm{~mm}$ to differentiate between plaque erosion and other plaque types is $\sim 400$ (12). By assessing plaque cavity, convex calcium, and maxLCBI4mm, NIRS-IVUS can accurately identify plaque rupture (sensitivity $=97 \%$ and specificity $=96 \%$ ), plaque erosion (sensitivity $=93 \%$ and specificity $=99 \%$ ), and calcified nodule (sensitivity $=100 \%$ and specificity $=99 \%)(13)$.

\section{MULTIMODALITY IMAGING}

Information obtained from multiple imaging techniques can complement each other. In fact, in addition to morphological evaluation, plaque tissue characterization is useful for differentiating plaque rupture, plaque erosion, and calcified 


\section{Plaque rupture}

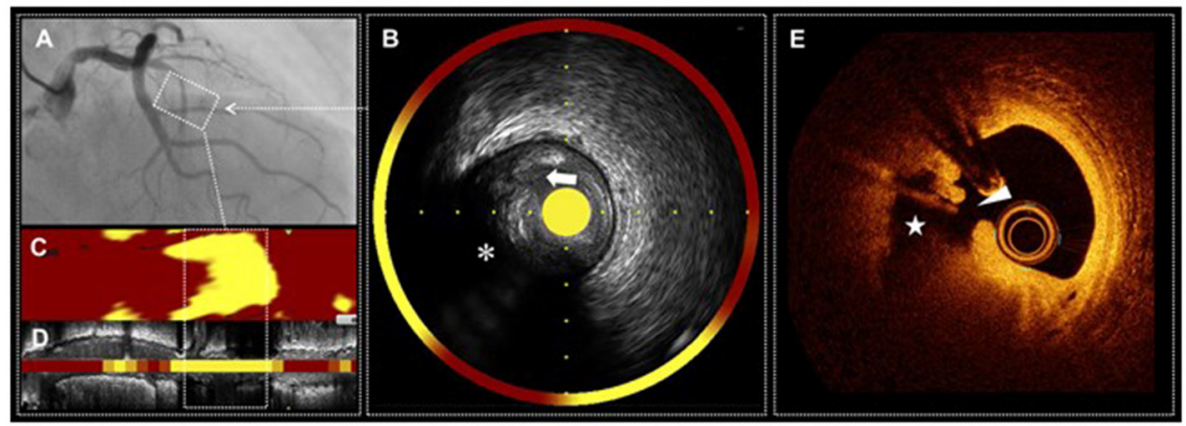

\section{Plaque erosion}

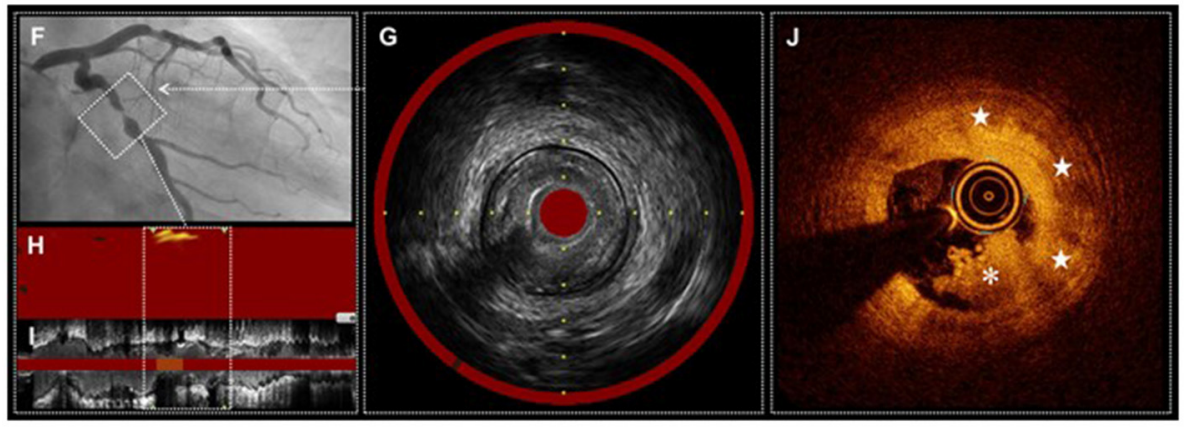

III. Calcified nodule

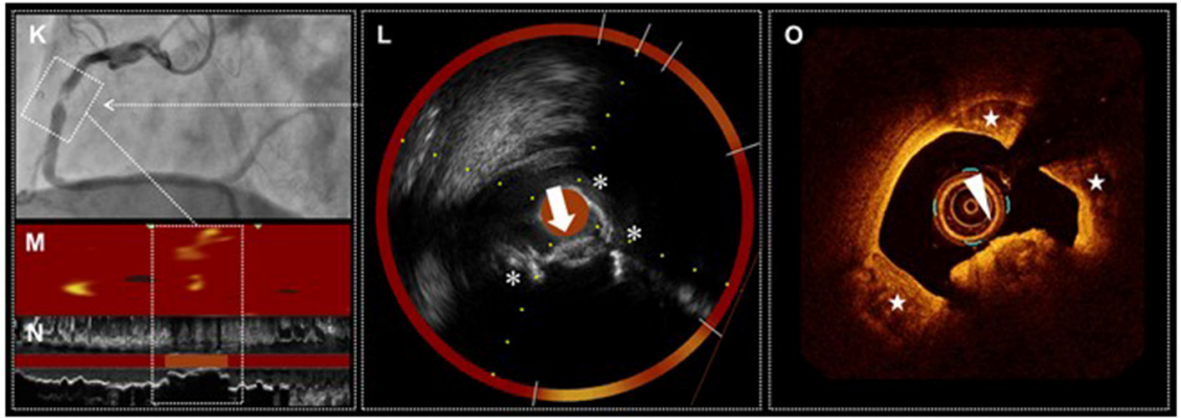

FIGURE 1 | Multimodality imaging of plaque rupture, plaque erosion, and calcified nodule. Plaque rupture (I) Angiogram shows occlusion of proximal LAD, the culprit lesion of STEMI (A). IVUS demonstrates ulceration (arrow) with a recess on the surface of attenuated plaque (asterisk) (B,D). NIRS identifies a large lipid content ( $m a x L C B I 4 m m=920)(B-D)$. OCT reveals plaque rupture characterized by a fibrous cap disruption (arrowhead) and a cavity (star) formation inside the plaque (E). Plaque erosion (II) Angiogram shows severe stenosis in mid LCX, the culprit lesion of STEMI (F). IVUS demonstrates the absence of plaque ulceration (G,I). NIRS identifies a small lipid content (maxLCBI4mm = 129) $(\mathbf{G}-\mathbf{I})$. OCT reveals plaque erosion characterized by the presence of attached thrombus (asterisk) overlying an intact fibrotic plaque (stars) (J). Calcified nodule (II) Angiogram shows severe stenosis and intraluminal filling defect in mid RCA, the culprit lesion of non-STEMI (K). IVUS demonstrates a convex calcium with irregular surface (arrow) and large superficial calcium (asterisks) (L,N). NIRS identifies a moderate lipid content $(\operatorname{maxLCBI} 4 \mathrm{~mm}=208)(\mathbf{L}-\mathbf{N})$. OCT reveals calcified nodule characterized by a protruding calcium with thrombi (arrowhead) and large superficial calcium (stars) (0). IVUS, intravascular ultrasound; LAD, left anterior descending artery; LCBI, lipid core burden index; LCX, left circumflex artery; NIRS, near-infrared spectroscopy; OCT, optical coherence tomography; RCA, right coronary artery; STEMI, ST-segment elevation myocardial infarction.

nodule (Table 1). Plaque rupture is identified by fibrous cap disruption and intra-plaque cavity in IVUS/OCT, attenuated plaque in IVUS, large lipid plaque in OCT, and high maxLCBI4mm $(>400)$ in NIRS (Figure 1-I). Plaque erosion is identified by intact fibrous cap and fibrotic plaque in OCT, and low maxLCBI4mm $(<400)$ in NIRS (Figure 1-II). Calcified nodule is identified by convex calcium and large calcium sheet in IVUS/OCT, and intermediate maxLCBI4mm in NIRS (Figure 1-III).

\section{IMPACT ON TREATMENT STRATEGY}

Information from multiple imaging techniques influence percutaneous coronary intervention (PCI) strategies and risk 
stratification in ACS. Plaque rupture is at high risk of slow flow or no-reflow, distal embolization, and microvascular obstruction associated with PCI (14-16). This is because the lesions with plaque rupture are rich in thrombi and lipid cores that can be embolic sources. A prospective randomized controlled trial using IVUS showed that the use of distal protection devise was effective in preventing slow flow during PCI for attenuated plaques (17). Plaque erosion has a better prognosis after PCI compared with plaque rupture $(18,19)$. Non-stent strategy may be an option for plaque erosion if sufficient lumen is obtained by thrombus aspiration alone. A prospective observational OCT study demonstrated that a majority $(92.5 \%)$ of patients with ACS caused by plaque erosion managed with dual antiplatelet therapy without stenting remained free of major adverse cardiovascular event during 1-year follow-up period (20). Calcified nodule is associated with stent underexpansion that is a well-known predictor of poor clinical outcome. An OCT-guided PCI registry disclosed that the minimum stent area at post-PCI was significantly smaller in calcified nodule than in plaque rupture and plaque erosion, with half of calcified nodule having a stent expansion index (calculated by minimum stent area / reference lumen area $\mathrm{x} 100$ ) of $<80 \%$ (21). Further research is needed to evaluate the effectiveness of more aggressive procedures such as rotational atherectomy, excimer laser coronary angioplasty, and shockwave intravascular lithotripsy for calcified nodules.

\section{LIMITATIONS}

Each intravascular imaging technique has its inherent limitations. IVUS has a coarse-resolution that is insufficient to detect ruptured thin fibrous caps and small ulcers on plaque surface. OCT has a small field of view and shallow imaging depth that often preclude assessment of the plaque burden and remodeling pattern. NIRS is unable to identify fibrous caps, in addition to its lack of morphological evaluation ability.

\section{REFERENCES}

1. Virmani R, Burke AP, Farb A, Kolodgie FD. Pathology of the vulnerable plaque. J Am Coll Cardiol. (2006) 47:C13-8. doi: 10.1016/j.jacc.2005.10.065

2. Johnson TW, Räber L, di Mario C, Bourantas C, Jia H, Mattesini A, et al. Clinical use of intracoronary imaging. Part 2: acute coronary syndromes, ambiguous coronary angiography findings, and guiding interventional decision-making: an expert consensus document of the European Association of Percutaneous Cardiovascular Interventions. Eur Heart J. (2019) 40:256684. doi: 10.1093/eurheartj/ehz332

3. Mintz GS, Nissen SE, Anderson WD, Bailey SR, Erbel R, Fitzgerald PJ, et al. American college of cardiology clinical expert consensus document on standards for acquisition, measurement and reporting of Intravascular Ultrasound Studies (IVUS). A report of the American College of Cardiology Task Force on Clinical Expert Consensus Documents. J Am Coll Cardiol. (2001) 37:1478-92. doi: 10.1016/s0735-1097(01)01175-5

4. Saito Y, Kobayashi Y, Fujii K, Sonoda S, Tsujita K, Hibi K, et al. Clinical expert consensus document on standards for measurements and assessment of intravascular ultrasound from the Japanese Association of Cardiovascular Intervention and Therapeutics. Cardiovasc Interv Ther. (2020) 35:1-12. doi: 10.1007/s12928-019-00625-6

\section{FUTURE PERSPECTIVES}

To overcome the limitations of each imaging technique, new efforts have been developed for data fusion methodologies and designs for hybrid dual-probe catheters. Combined IVUS-OCT imaging provides a fusional image of OCT with a high resolution for the plaque surface and IVUS with a large imaging depth (22). Combined IVUS-OCT imaging enables a comprehensive depiction of coronary atherosclerotic plaque. Combined NIRSOCT imaging allows the detection of lipid core by spectroscopy and structural features, including cap thickness, by OCT (23). Combined NIRS-OCT imaging will facilitate the identification of thin-capped fibroatheroma, which is recognized as a precursor to plaque rupture.

\section{CONCLUSION}

At this time, there is no single imaging technique that is perfect for evaluating atherosclerosis. The combination of multiple imaging techniques provides a lot of information about the morphology and composition of atherosclerosis. Detailed coronary evaluation with multiple imaging techniques allows a better understanding of the pathology of ACS and guides lesion-specific treatment.

\section{AUTHOR CONTRIBUTIONS}

TK wrote the manuscript. KT, YI, and YS collected images of IVUS, OCT, and NIRS. ST, T-PT, YC, and D-WP reviewed and revised the manuscript. All authors approved the final manuscript.

\section{FUNDING}

This work was partially supported by JSPS KAKENHI Grant Number JP19K12846.

5. Wu X, Mintz GS, Xu K, Lansky AJ, Witzenbichler B, Guagliumi G, et al. The relationship between attenuated plaque identified by intravascular ultrasound and no-reflow after stenting in acute myocardial infarction: the HORIZONS-AMI (Harmonizing Outcomes With Revascularization and Stents in Acute Myocardial Infarction) trial. JACC Cardiovasc Interv. (2011) 4:495-502. doi: 10.1016/j.jcin.2010.12.012

6. Lee JB, Mintz GS, Lisauskas JB, Biro SG, Pu J, Sum ST, et al. Histopathologic validation of the intravascular ultrasound diagnosis of calcified coronary artery nodules. Am J Cardiol. (2011) 108:1547-51. doi: 10.1016/j.amjcard.2011.07.014

7. Fujii K, Kubo T, Otake H, Nakazawa G, Sonoda S, Hibi K, et al. Expert consensus statement for quantitative measurement and morphological assessment of optical coherence tomography. Cardiovasc Interv Ther. (2020) 35:13-8. doi: 10.1007/s12928-019-00626-5

8. Kubo T, Imanishi T, Takarada S, Kuroi A, Ueno S, Yamano T, et al. Assessment of culprit lesion morphology in acute myocardial infarction: ability of optical coherence tomography compared with intravascular ultrasound and coronary angioscopy. J Am Coll Cardiol. (2007) 50:9339. doi: 10.1016/j.jacc.2007.04.082

9. Tearney GJ, Regar E, Akasaka T, Adriaenssens T, Barlis P, Bezerra HG, et al. Consensus standards for acquisition, measurement, and reporting 
of intravascular optical coherence tomography studies: a report from the international working group for intravascular optical coherence tomography standardization and validation. J Am Coll Cardiol. (2012) 59:105872. doi: 10.1016/j.jacc.2011.09.079

10. Kubo T, Tanaka A, Ino Y, Kitabata H, Shiono Y, Akasaka T. Assessment of coronary atherosclerosis using optical coherence tomography. J Atheroscler Thromb. (2014) 21:895-903. doi: 10.5551/jat.25452

11. Jia $\mathrm{H}$, Abtahian F, Aguirre AD, Lee S, Chia S, Lowe H, et al. In vivo diagnosis of plaque erosion and calcified nodule in patients with acute coronary syndrome by intravascular optical coherence tomography. J Am Coll Cardiol. (2013) 62:1748-58. doi: 10.1016/j.jacc.2013.05.071

12. Madder RD, Goldstein JA, Madden SP, Puri R, Wolski K, Hendricks M, et al. Detection by near-infrared spectroscopy of large lipid core plaques at culprit sites in patients with acute ST-segment elevation myocardial infarction. JACC Cardiovasc Interv. (2013) 6:838-46. doi: 10.1016/j.jcin.2013.04.012

13. Terada K, Kubo T, Kameyama T, Matsuo Y, Ino Y, Emori H, et al. NIRS-IVUS for differentiating coronary plaque rupture, erosion, and calcified nodule in acute myocardial infarction. JACC Cardiovasc Imaging. (2021) 14:144050. doi: 10.1016/j.jcmg.2020.08.030

14. Shiono Y, Kubo T, Tanaka A, Tanimoto T, Ota S, Ino Y, et al. Impact of attenuated plaque as detected by intravascular ultrasound on the occurrence of microvascular obstruction after percutaneous coronary intervention in patients with ST-segment elevation myocardial infarction. JACC Cardiovasc Interv. (2013) 6:847-53. doi: 10.1016/j.jcin.2013.01.142

15. Tanaka A, Imanishi $T$, Kitabata $H$, Kubo $T$, Takarada $S$, Tanimoto $T$, et al. Lipid-rich plaque and myocardial perfusion after successful stenting in patients with non-ST-segment elevation acute coronary syndrome: an optical coherence tomography study. Eur Heart J. (2009) 30:134855. doi: 10.1093/eurheartj/ehp122

16. Terada K, Kubo T, Madder RD, Ino Y, Takahata M, Shimamura K, et al. Nearinfrared spectroscopy to predict microvascular obstruction after primary percutaneous coronary intervention. EuroIntervention. (2021) 17:e999e1006. doi: 10.4244/EIJ-D-20-01421

17. Hibi K, Kozuma K, Sonoda S, Endo T, Tanaka H, Kyono H, et al. A Randomized study of distal filter protection versus conventional treatment during percutaneous coronary intervention in patients with attenuated plaque identified by intravascular ultrasound. JACC Cardiovasc Interv. (2018) 11:1545-55. doi: 10.1016/j.jcin.2018.03.021

18. Niccoli G, Montone RA, Di Vito L, Gramegna M, Refaat H, Scalone G, et al. Plaque rupture and intact fibrous cap assessed by optical coherence tomography portend different outcomes in patients with acute coronary syndrome. Eur Heart J. (2015) 36:1377-84. doi: 10.1093/eurheartj/ehv029
19. Yonetsu T, Lee T, Murai T, Suzuki M, Matsumura A, Hashimoto Y, et al. Plaque morphologies and the clinical prognosis of acute coronary syndrome caused by lesions with intact fibrous cap diagnosed by optical coherence tomography. Int J Cardiol. (2016) 203:766-74. doi: 10.1016/j.ijcard.2015. 11.030

20. Xing L, Yamamoto E, Sugiyama T, Jia H, Ma L, Hu S, et al. EROSION Study (effective anti-thrombotic therapy without stenting: intravascular optical coherence tomography-based management in plaque erosion): a 1-year follow-up report. Circ Cardiovasc Interv. (2017) 10:e005860. doi: 10.1161/CIRCINTERVENTIONS.117.0 05860

21. Khalifa AKM, Kubo T, Ino Y, Terada K, Emori H, Higashioka D, et al. Optical coherence tomography comparison of percutaneous coronary intervention among plaque rupture, erosion, and calcified nodule in acute myocardial infarction. Circ J. (2020) 84:911-6. doi: 10.1253/circj.CJ-20-0014

22. Ono M, Kawashima H, Hara H, Gao C, Wang R, Kogame N, et al Advances in IVUS/OCT and future clinical perspective of novel hybrid catheter system in coronary imaging. Front Cardiovasc Med. (2020) 7:119. doi: $10.3389 /$ fcvm. 2020.00119

23. Bourantas CV, Jaffer FA, Gijsen FJ, van Soest G, Madden SP, Courtney BK, et al. Hybrid intravascular imaging: recent advances, technical considerations, and current applications in the study of plaque pathophysiology. Eur Heart $J$. (2017) 38:400-12. doi: 10.1093/eurheartj/ehw097

Conflict of Interest: The authors declare that the research was conducted in the absence of any commercial or financial relationships that could be construed as a potential conflict of interest.

Publisher's Note: All claims expressed in this article are solely those of the authors and do not necessarily represent those of their affiliated organizations, or those of the publisher, the editors and the reviewers. Any product that may be evaluated in this article, or claim that may be made by its manufacturer, is not guaranteed or endorsed by the publisher.

Copyright (c) 2022 Kubo, Terada, Ino, Shiono, Tu, Tsao, Chen and Park. This is an open-access article distributed under the terms of the Creative Commons Attribution License (CC BY). The use, distribution or reproduction in other forums is permitted, provided the original author $(s)$ and the copyright owner(s) are credited and that the original publication in this journal is cited, in accordance with accepted academic practice. No use, distribution or reproduction is permitted which does not comply with these terms. 